07

\title{
Рекомбинационные процессы в преобразователях энергии источников $\beta$-излучения в электрическую энергию
}

\author{
(C) И.Е. Абанин, В.В. Амеличев, С.В. Булярский, А.В. Лакалин \\ „Научно-производственный комплекс «Технологический центр» МИЭт“, \\ 124498 Москва, Зеленоград, Россия \\ e-mail: bulyar2954@mail.ru
}

(Поступило в Редакцию 21 января 2016 г.)

Исследованы преобразователи $\beta$-излучения в электрический ток, изготовленные на основе высокоомного кремния, а также процессы, обусловливающие эффективность преобразования. Показано, что последняя величина обусловлена рекомбинацией в области пространственного заряда преобразователей при малых уровнях инжекции. Разработана методика определения рекомбинационных параметров центров, которая опирается на новые алгоритмы преобразования, переводящие монотонную и в целом экспоненциальную вольт-амперную характеристику в кривую с особенностями, позволяющими определить параметры центров рекомбинации. Рассчитаны параметры данных центров в широком диапазоне температур и выявлены области в координатах температур - напряжение прямого смещения, где рекомбинационные потоки перехватываются тем или иным центром рекомбинации, что оказывает влияние на величину тока насыщения вольт-амперной характеристики и эффективность преобразования.

\section{Введение}

Прямое преобразование энергии радиоактивных изотопов заключается в том, что испускаемые ими $\beta$-электроны генерируют в полупроводнике электроннодырочные пары, которые разделяются электрическим полем $p-n$-перехода или контакта металл-полупроводник $[1-8]$. Возникает $\beta$-вольтаический эффект. Этот эффект является аналогом фотоэлектрического с той разницей, что образование электрон-дырочных пар в кристаллической решетке полупроводника происходит под воздействием быстрых электронов, а не фотонов. Единичный элемент $\beta$-электрического преобразователя представляет собой полупроводник с $p-n$ или $p-i-n$-переходом, контактирующий с радиоактивным источником $\beta$-излучения. Образование электрондырочных пар происходит при взаимодействии проникших в объем полупроводника $\beta$-частиц с электронами атомов кристаллической решетки. Количество образовавшихся неравновесных носителей заряда в объеме полупроводника растет с энергией $\beta$-электронов и интенсивностью их потока.

В научной литературе по $\beta$-вольтаическим источникам питания [1-8] ищутся пути повышения эффективности преобразования. Одним из направлений достижения этой цели является снижение рекомбинационных потерь. Далеко не все электронно-дырочные пары вносят вклад в величину тока, генерируемого $\beta$-электронами. В $i$-слое структуры наряду с процессом генерации идет альтернативный процесс - рекомбинации. В результате этого часть носителей заряда теряется. Для того чтобы оценить данные потери, необходимо исследовать изменение времени жизни и связанные с ним причины возникновения рекомбинационных токов. Поэтому механизмы рекомбинации в таких структурах должны быть понятны, для того чтобы определить пути снижения рекомбинационных потерь.

Процессы генерации-рекомбинации определяют величину „темновых“ токов, которые в научной литературе называют также токами насыщения. Увеличение этих токов также приводит к снижению эффективности преобразования тока (коэффициента полезного действия батареи - $\eta$ ), генерированного электронами высоких энергий. Выражение для коэффициента преобразования хорошо известно как для солнечных элементов [9], так и для ядерных батарей [10]:

$$
\eta=\frac{I_{s c} \chi k T}{e P_{\beta}}\left[\ln \left(\frac{I_{s c}}{I_{s}}\right)+1\right],
$$

где $I_{s c}-$ ток короткого замыкания, $P_{\beta}-$ мощность, излучаемая $\beta$-источником, $\chi-$ коэффициент. Данный коэффициент зависит от вида вольт амперной характеристики и нагрузочного сопротивления. Он показывает отклонение максимальной электрической мощности, выделяемой батареей, от произведения $-I_{s c} U_{x x}$. Как правило, $0.5<\chi<0.8$. $I_{s}-$ ток насыщения, который определяется механизмами переноса свободных носителей заряда, формирующими прямую и обратную вольтамперную характеристику.

Целью настоящей работы является изучение механизмов, формирующих времена жизни электронов и дырок, а также величину тока насыщения преобразователей энергии, выявление рекомбинационных центров, ответственных за этот процесс. Все указанные величины оказывают влияние на эффективность преобразования. В работе использована новая методика определения характеристик центров рекомбинации, которая проста, удобна и может быть полезна широкому кругу исследователей. 


\section{Методика эксперимента и экспериментальные результаты}

Преобразователи были изготовлены на базе $p-i-n$ переходов, с толщиной $i$-области порядка $100 \mu \mathrm{m}$. Такая толщина слоя выбиралась из соображений универсальности, так как в качестве $\beta$-источников в дальнейшем будут применяться изотопы с разной энергией электронов от 3 до $160 \mathrm{keV}$. Структуры изготавливались методом планарной технологии на высокоомном кремнии. Их рабочая площадь составляла $1 \mathrm{~cm}^{2}$. Основной методикой эксперимента является измерение вольтамперных характеристик. Измерения выполнены на автоматизированной установке, укомплектованной высокоточными измерительными приборами. Вольт-амперные характеристики исследуемых полупроводниковых приборов воспроизводились с высокой точностью как при прямом, так и при обратном смещении, что характерно для изделий, полученных групповым методом на одной пластине кремния.

На рис. 1. приведен дифференциальный коэффициент наклона типичной вольт-амперной характеристики исследуемых преобразователей, вычисленный путем численного дифференцирования:

$$
\beta_{r}=\frac{q}{k T}\left(\frac{d \ln j_{r}}{d U}\right)^{-1}=\frac{q j_{r}}{k T}\left(\frac{d j_{r}}{d U}\right)^{-1}
$$

где $j_{r}-$ плотность тока $p-i-n$-диода, $q-$ заряд электрона, $U-$ напряжение прямого смещения.

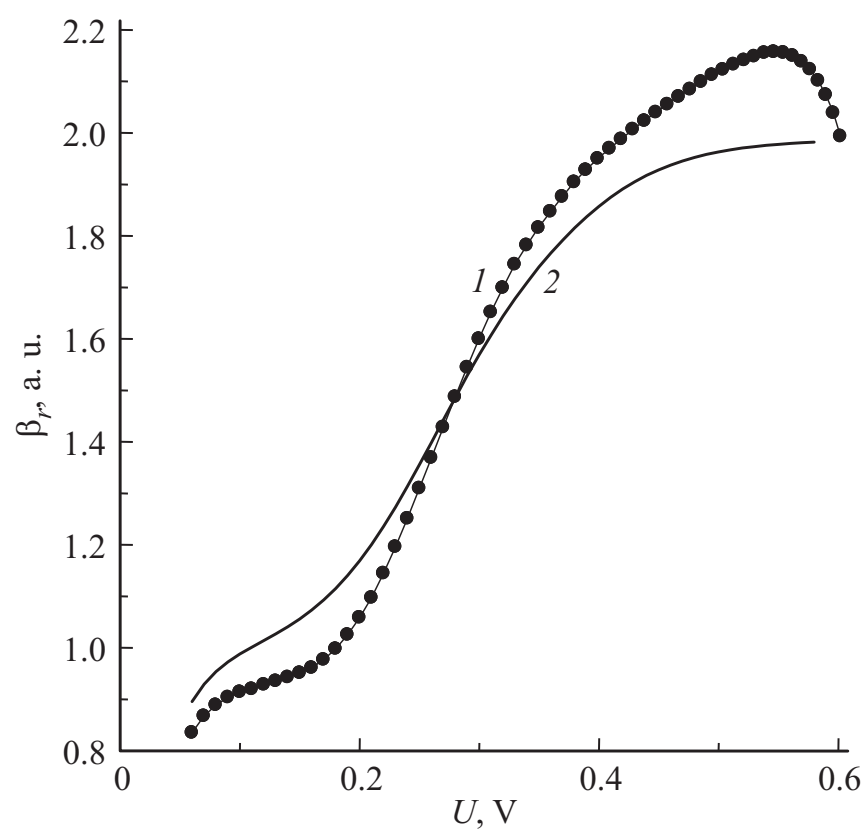

Pис. 1. Дифференциальный показатель наклона и его восстановление, по рассчитанным в работе коэффициентам центров рекомбинации. 1 - численный расчет из эксперимента по формуле (2), 2 - расчет по формуле работы [13].

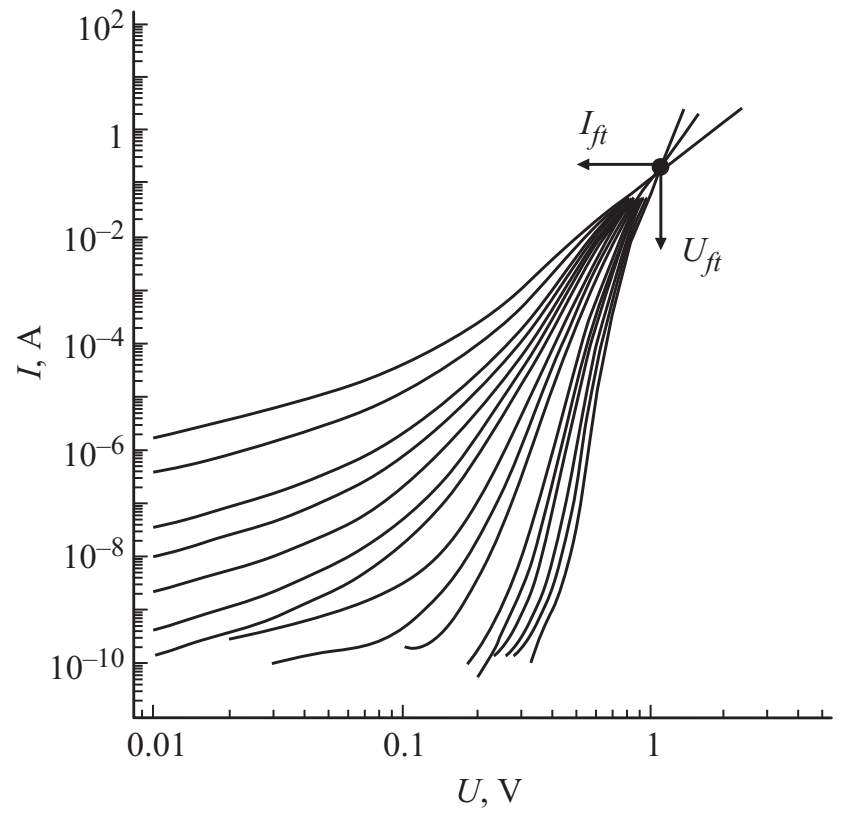

Рис. 2. Вольт-амперные характеристики $p-i-n$-диодов в двойном логарифмическом масштабе измеренных при температурах, K: слева направо меняется через $20 \mathrm{~K}$ от 373 до 233.

Дифференциальный показатель наклона позволяет качественно охарактеризовать механизмы протекания тока [11]. Так как эта величина больше единицы практически во всем диапазоне измеряемых напряжений, то можно сделать заключение, что диффузионный ток в исследуемых структурах места не имеет. В области напряжений прямого смещения $0.1<U<0.4 \mathrm{~B}$ значения этого коэффициента плавно возрастают от 1 до 2 . Такое поведение дифференциального показателя наклона характерно для токов рекомбинации в области пространственного заряда на глубоких рекомбинационных центрах. При больших напряжениях этот показатель превышает значение 2, что может быть обусловлено наступлением режима высокого уровня инжекции.

В области напряжений прямого смещения, больших $0.4 \mathrm{~V}$, вольт-амперные характеристики, перестроенные в координатах $\sqrt{I}=f(U)$, позволяют найти величину диффузионного потенциала [11]. В нашем случае он равен $0.48 \mathrm{~V}$. Это значение порядка половины запрещенной зоны кремния, что характерно для наличия в диоде высокоомной области.

На рис. 2 показаны вольт-амперные характеристики исследуемых преобразователей, построенные в двойном логарифмическом масштабе. При высоких напряжениях прямого смещения продолжения данных характеристик пересекаются в одной точке. Это характерно для токов, ограниченных пространственным зарядом. Точка пересечения вольт-амперных характеристик связана с полным заполнением ловушек внутри высокоомной области преобразователя. Напряжение полного заполнения ловушек позволяет оценить концентрацию ловушек в 
высокоомном слое [11]:

$$
j_{f t}=e \mu_{n} N_{d} \frac{U_{f t}}{W}, \quad U_{f t}=\frac{e W N_{t}}{\varepsilon_{0} \varepsilon_{S}},
$$

где $j_{f t}-$ плотность тока в точке полного заполнения ловушек, $N_{d}-$ концентрация остаточных мелких примесей, $U_{f t}$ - напряжение полного заполнения ловушек, $W-$ ширина базы диода, $N_{t}$ - концентрация глубоких ловушек, $\varepsilon_{0}-$ диэлектрическая постоянная вакуума, $\varepsilon_{S}-$ статическая диэлектрическая постоянная полупроводника.

Расчеты по формуле (3) дали следующие результаты: концентрация глубоких центров $-1.4 \cdot 10^{11} \mathrm{~cm}^{-3}$, подвижность носителей заряда $2800 \mathrm{~V} \mathrm{~cm} / \mathrm{s}$.

Удельное сопротивление, которое оценивалось по предварительным измерениям на кремниевой пластине, а затем по сопротивлению базы прибора, имеет величину порядка $500 \Omega \mathrm{cm}$, что соответствует собственному кремнию. Концентрация носителей заряда при комнатной температуре порядка $10^{10} \mathrm{~cm}^{-3}[9]$. Таким образом, концентрация глубоких ловушек превышает концентрацию свободных носителей заряда. Именно поэтому стало возможным наблюдение токов, ограниченных пространственным зарядом. Такое соотношение концентраций технологических примесей и дефектов приводит к не типичному поведению электрических свойств диодов.

Приведенные результаты показывают, что интересующие нас величины, связанные с рекомбинационными свойствами: время жизни и темновой ток рекомбинации, определяющий эффективность преобразования, необходимо определять при малых уровнях смещения до $0.4 \mathrm{~V}$.

\section{Анализ рекомбинационных процессов при малом уровне инжекции}

Научные результаты, полученные другими авторами $[2,3,7,8]$, а также данные предварительных экспериментов, показывают, что величина напряжения холостого хода у $\beta$-преобразователей с источником $\mathrm{Ni}-63$, не превышает $0.15 \mathrm{~V}$. Поэтому рабочая область напряжений на преобразователях лежит в диапазоне малого уровня инжекции, для которого характерны процессы рекомбинации в области пространственного заряда. Выражения для плотности тока рекомбинации в ОПЗ $p-n$-перехода, получены в работах [11-13]. Этими же авторами разработана методика, которая носит название рекомбинационная спектроскопия. В настоящей работе модель рекомбинационной спектроскопии получила свое дальнейшее развитие.

В случае нескольких глубоких центров результирующий ток представляет сумму токов рекомбинации через каждый из них [11-13]:

$$
I_{r}=\sum_{m=1}^{g} \frac{q S w(U) c_{n m} c_{p m} n_{i}^{2}\left(e^{q U / k T}-1\right) N_{t m}}{2 n_{i} \sqrt{c_{n m} c_{p m}} e^{q U / 2 k T}+c_{n m} n_{1 m}+c_{p m} p_{1 m}} \frac{2 k T}{q\left(V_{d}-U\right)},
$$

где $w$ - ширина ОПЗ, $g$ - число двухзарядных рекомбинационных центров, одновременно участвующих в процессе рекомбинации, $q-$ заряд электрона, $S-$ площадь диода, $c_{n m}$ - коэффициент захвата электронов из зоны проводимости рекомбинационным центром с номером $m, C_{p m}-$ коэффициент захвата дырок из зоны проводимости рекомбинационным центром с номером $m, U$ - напряжение смещения на области пространственного заряда диода, $k-$ постоянная Больцмана, $N_{t m}$ - концентрация центров рекомбинации одного типа с номером $m, n_{i}$ - концентрация электронов в собственном кремнии, $n_{1 m}-$ концентрация электронов $\left(p_{1 m}\right.$-дырок) в зоне проводимости (валентной зоне) при условии, что уровень Ферми совпадает с уровнем энергии рекомбинационного центра с номером $m$ :

$$
\begin{aligned}
& n_{1 m}=N_{c} \exp \left(-\frac{E_{c}-E_{t m}}{k T}\right), \\
& p_{1 m}=N_{v} \exp \left(-\frac{E_{t m}-E_{v}}{k T}\right),
\end{aligned}
$$

где $N_{c}$ - эффективная плотность состояний зоны проводимости, $N_{v}$ - эффективная плотность состояний валентной зоны, $E_{t m}$ - энергия активации глубокого рекомбинационного центра, $E_{c}$ - энергия дна зоны проводимости, $E_{v}$ - энергия потолка валентной зоны.

Для разработки алгоритма вычисления параметров центров представим плотность рекомбинационного тока в области пространственного заряда в более простом виде для одного рекомбинационного центра

$$
j_{r}=\frac{2 k T w(U)}{\left(V_{d}-U\right)} \frac{c_{n} c_{p} n_{i}^{2} N_{t}\left[\exp \left(\frac{q U}{k T}\right)-1\right]}{2 n_{i} \sqrt{c_{n} c_{p}} \exp \left(\frac{q U}{2 k T}\right)+c_{n} n_{1}+c_{p} p_{1}} .
$$

Величина $V_{d}$ нами уже определена по вольт-амперной характеристике, зависимость $w(U)$ находим из емкостных измерений, тогда неизвестными остаются четыре параметра: $c_{n}-$ коэффициент захвата электронов из зоны проводимости рекомбинационным центром, $c_{p}-$ коэффициент захвата дырок из валентной зоны рекомбинационным центром, $E_{m}$ - энергия активации глубокого рекомбинационного центра, $N_{t}$ - концентрация центров рекомбинации.

Заметим, что произведения $c_{n} N_{t}=\tau_{n 0}^{-1}, c_{p} N_{t}=\tau_{p 0}^{-1}$ определяют величины, которые являются временем жизни при захвате электрона на пустой рекомбинационный центр и временем жизни при захвате дырки на заполненный электроном центр рекомбинации. Эти времена являются характеристическими величинами для центров рекомбинации. Преобразуем (6) путем деления на $n_{i}$; $\frac{2 k T w(U)}{\left(V_{d}-U\right)}$ и $\exp \left(\frac{q U}{2 k T}\right)-1$, получаем, а также используем определенные выше времена жизни:

$$
R_{p r}=\frac{\frac{\tau_{n 0}^{-1} \tau_{p 0}^{-1}}{N_{t}} n_{i}\left[\exp \left(\frac{q U}{2 k T}\right)+1\right]}{\frac{2 n_{i} \sqrt{\tau_{n 0}^{-1} \tau_{p 0}^{-1}}}{N_{t}} \exp \left(\frac{q U}{2 k T}\right)+c_{n} n_{1}} .
$$

Величину, определяемую формулой (7), назовем приведенной скоростью рекомбинации. Эта величина характеризует рекомбинационный поток через уровень рекомбинации и является обратной к времени жизни. Величины $\tau_{n 0}$ и $\tau_{p 0}$ отличаются не более, чем в 10 раз. Уровень рекомбинационного центра, как правило, не лежит точно в середине зоны. 
Пусть этот уровень выше середины зоны на величину $3 k T$ и расположен ближе к зоне проводимости, тогда $\left(E_{c}-E_{t}\right)-\left(E_{t}-E_{v}\right)=6 k T$. Отношение $n_{1} / p_{1} \approx$ $\approx e^{6}=414$. Даже при неудовлетворительном отношении коэффициентов захвата $c_{n} n_{1} \gg c_{p} p_{1}$. Поэтому последнее произведение в формуле (7) опущено.

Соотношение членов в знаменателе изменяется с увеличением приложенного напряжения. При некотором напряжении $U_{0}$ первое и второе слагаемые в знаменателе станут равными. При этом напряжении можно вычислить энергию активации рекомбинационного центра

$$
E_{c}-E_{t}=E_{g} / 2-e U_{0} / 2+\frac{3}{4} k T \ln \left(\frac{m_{n}^{*}}{m_{p}^{*}}\right)+\frac{1}{2} k T \ln \left(\frac{\tau_{p 0}}{\tau_{n 0}}\right) .
$$

Энергия активации глубокого центра определяется с точностью до последнего слагаемого, поскольку отношение $\tau_{p 0} / \tau_{n 0}$, как правило, неизвестно. Для отношения коэффициентов захвата 10 ошибка составляет $0.028 \mathrm{eV}$ при комнатной температуре. Величина третьего слагаемого -0.01 . Поэтому если обращать внимание на первые два слагаемых, то неопределенность в определении энергии активации составляет $0.02 \mathrm{eV}$, что является вполне допустимым для данного простого метода определения энергии.

Когда первое слагаемое в знаменателе формулы (7) намного меньше второго, то

$$
R_{p r S} \approx \tau_{n 0}^{-1} \tau_{p 0}^{-1} / 2
$$

Эта величина постоянна, она соответствует максимуму скорости рекомбинации. При обратном соотношении величин слагаемых в знаменателе (7)

$$
R_{p r} \approx \frac{1}{\tau_{p 0}} \frac{n_{i}^{2}}{n_{1}}\left[\exp \left(\frac{q U}{2 k T}+1\right)\right] .
$$

Уравнения (7)-(10) являются основой для вычисления параметров рекомбинационного центра. Алгоритм вычислений следующий: по формуле (8) определяем в первом приближении энергию активации рекомбинационного центра. Выберем на зависимости приведенной скорости рекомбинации некоторую точку с координатами $R_{1}, U_{1}$; тогда, решая совместно систему уравнений (7) - (10), получаем для характеристических времен жизни следующие соотношения:

$$
\begin{aligned}
\tau_{n 0}^{-1} & =\frac{4 R_{p r S}^{2} n_{i}}{n_{1} R_{1}} \exp \left(\frac{U_{1}}{2 k T}\right), \\
\tau_{p 0}^{-1} & =\frac{n_{1} R_{1}}{n_{i}} \exp \left(-\frac{U_{1}}{2 k T}\right), \\
\frac{c_{p}}{c_{n}} & =\frac{n_{1}^{2} R_{1}^{2}}{4 R_{p r S}^{2} n_{i}^{2}} \exp \left(\frac{e U_{1}}{k T}\right) .
\end{aligned}
$$

Используя оценку концентрации рекомбинационных центров по токам, ограниченным пространственным зарядом, вычисляем коэффициенты захвата, а затем восстанавливаем дифференциальные коэффициенты вольтамперной характеристики. Соответствующие формулы

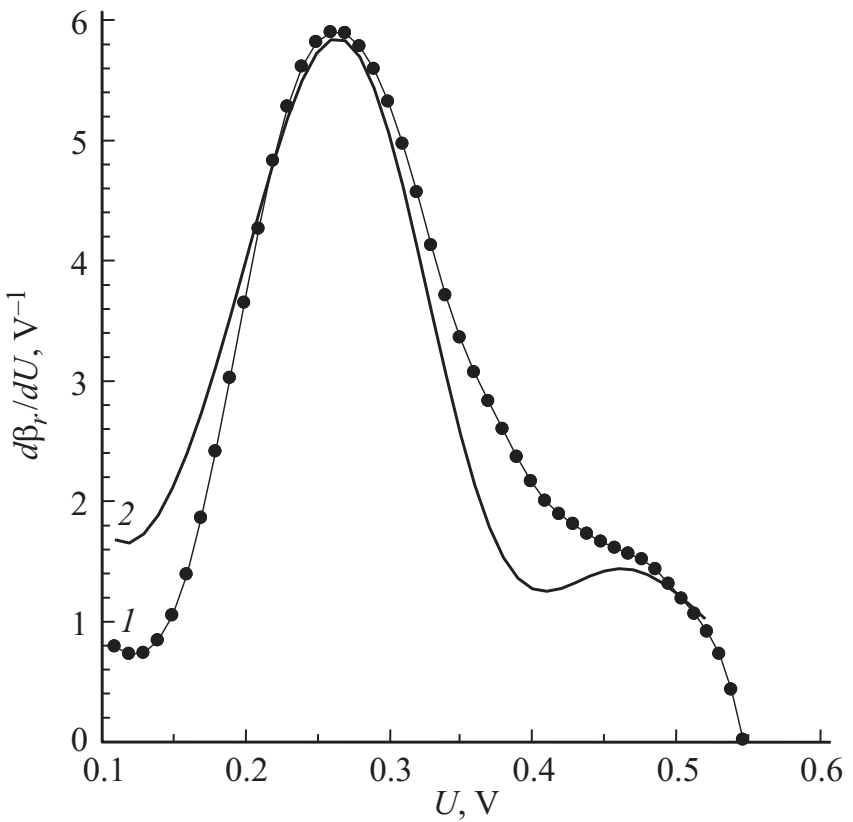

Рис. 3. Восстановление производной дифференциального показателя наклона по формулам работы [13] и рассчитанным коэффициентам: 1 - эксперимент, 2 - расчет.

приведены в работе [13]. Результат восстановления дифференциальных коэффициентов приведены на рис. 1 и 3. Удовлетворительное согласие экспериментальных и расчетных кривых указывает на достоверность проведенных вычислений.

Анализ экспериментальной кривой приведенной скорости рекомбинации позволяет разделить ее на отдельные рекомбинационные процессы. Методика разделения описана в работах $[11,13]$. В настоящей работе выделено три рекомбинационных центра с энергией активации $0.55,0.45,0.39 \mathrm{eV}$; расчетные параметры приведены в таблице.

В дальнейшем система уравнений (7)-(11) использовалась для анализа температурных зависимостей времени жизни. При каждом значении температуры было проведено разделение рекомбинационных процессов на составляющие и определены характеристические пара-

Параметры рекомбинационных центров при $T=295 \mathrm{~K}$

\begin{tabular}{l|c|c|c}
\hline $\begin{array}{l}\text { Энергия активации } \\
\text { электронов с рекомбина- } \\
\text { ционных центров, eV }\end{array}$ & 0.55 & 0.45 & 0.39 \\
\hline $\begin{array}{l}\text { Концентрация глубоких } \\
\text { рекомбинационных } \\
\text { центров, } \mathrm{cm}^{-3}\end{array}$ & $1.5 \cdot 10^{10}$ & $1.5 \cdot 10^{11}$ & $1.5 \cdot 10^{11}$ \\
\hline $\begin{array}{l}\text { Коэффициент захвата } \\
\text { для электронов, } \mathrm{cm}^{3} \mathrm{~s}^{-1}\end{array}$ & $6 \cdot 10^{-8}$ & $8 \cdot 10^{-8}$ & $3 \cdot 10^{-7}$ \\
\hline $\begin{array}{l}\text { Коэффициент захвата } \\
\text { дырок, } \mathrm{cm}^{3} \mathrm{~s}^{-1}\end{array}$ & $2 \cdot 10^{-8}$ & $8 \cdot 10^{-8}$ & $7 \cdot 10^{-8}$
\end{tabular}


метры рекомбинационных центров. Полученные результаты свидетельствуют о том, что рекомбинационные процессы в исследуемых структурах достаточно сильно зависят от температуры (рис. 4,5 ).

Скорость рекомбинации через центр рекомбинации с энергией $0.55 \mathrm{eV}$ выше и ниже при высоких и низких температурах. При средних температурах она минимальна и, следовательно, время жизни максимально. Эффективность преобразования была бы максимальна при комнатных температурах, если бы в рекомбинационном процессе участвовал только этот центр рекомбинации. Достаточно высокое время жизни, которое обусловле-

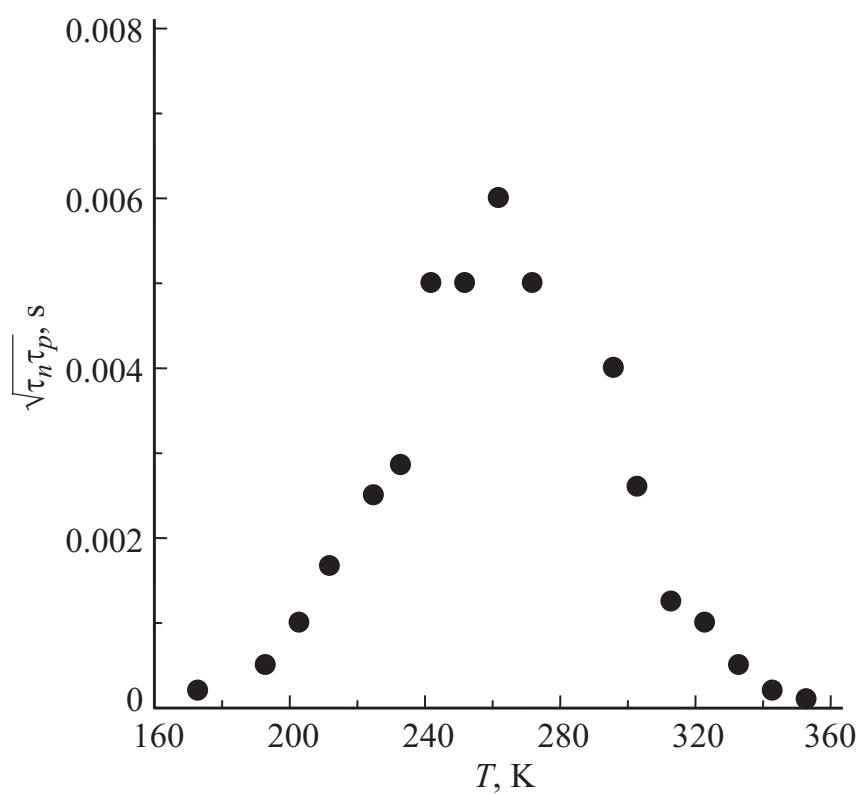

Рис. 4. Температурная зависимость времени жизни при рекомбинации через уровень с энергий активации $0.55 \mathrm{eV}$.

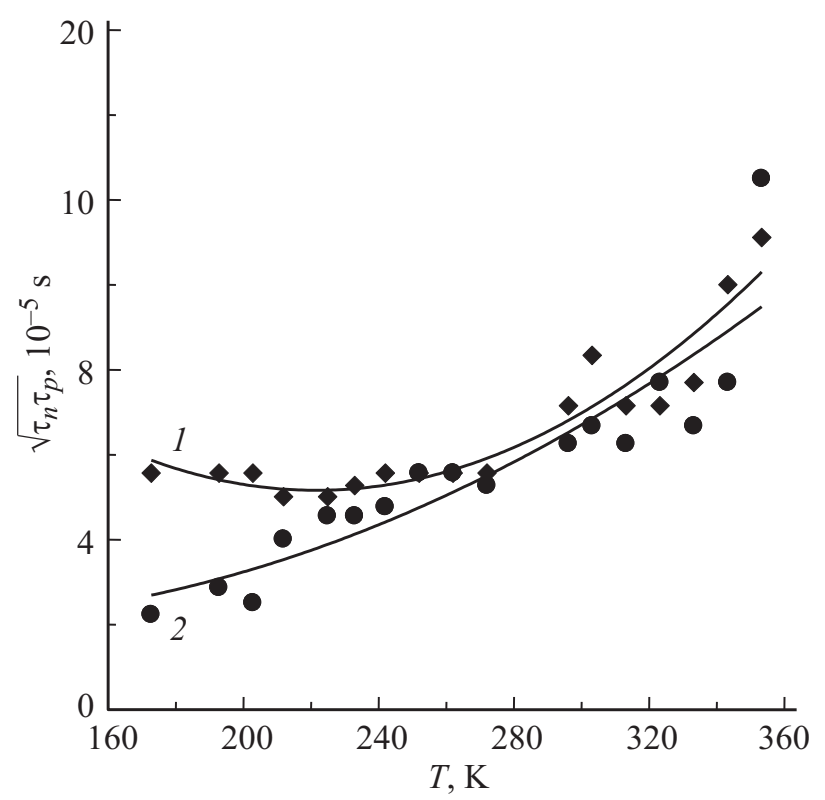

Рис. 5. Температурная зависимость времени жизни через уровни с энергией активации, $\mathrm{eV}: 0.45$ (1), 0.39 (2).

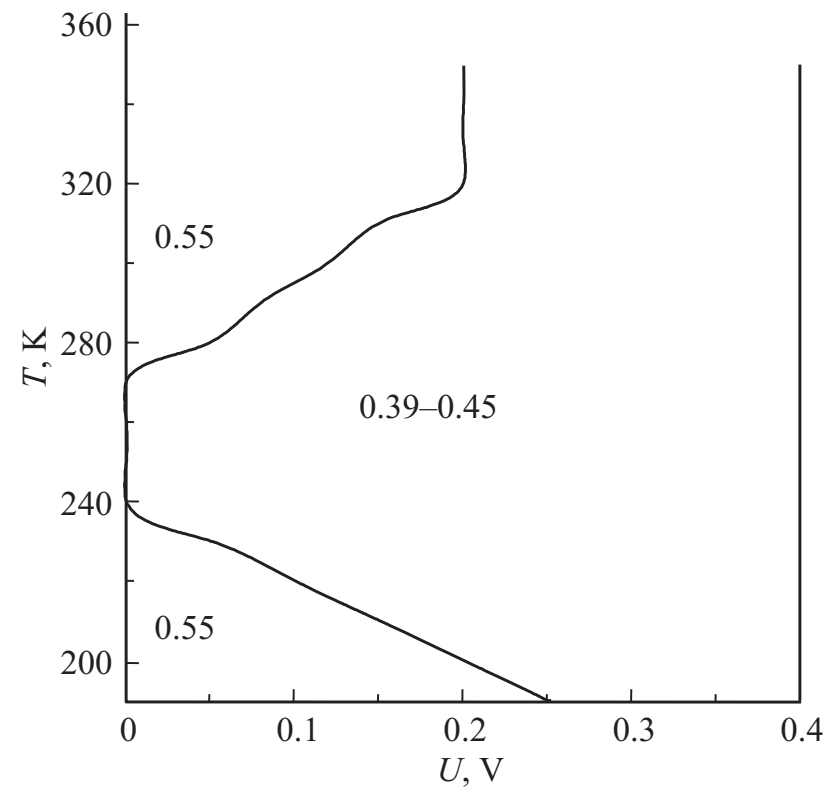

Рис. 6. Области в пространстве температур и прямого смещения, в которых преобладает рекомбинация через тот или иной центр. Энергии центров рекомбинации приведены на рисунке в $\mathrm{eV}$.

но данным центром, связано с низкой концентрацией, которая равна примерно $10^{10} \mathrm{~cm}^{-3}$. Возможно, что этот центр связан с остаточным легированием кремния золотом или железом.

Рекомбинационные центры с энергиями 0.45 и $0.39 \mathrm{eV}$ имеют достаточно стабильные параметры, которые слабо зависят от температуры. Время жизни, которое они формируют, также стабильно в широком интервале температур.

Рассмотрим, какие центры оказывают доминирующее влияние с изменением напряжения прямого смещения и температуры. При прямом смещении энергия активации тока в соответствии с формулой (6) изменяется по закону

$$
E_{a}=E_{g}-E_{t}-e U
$$

откуда

$$
E_{t}=E_{g}-E_{a}-e U .
$$

Для того, чтобы выделить области в пространстве напряжение прямого смещения-температура, в которых рекомбинационные потоки проходят через те либо иные центры, поступим следующим образом:

- построим температурные зависимости прямого тока при различных напряжениях прямого смещения в координатах Аррениуса,

- выделим на них прямолинейные участки и вычислим энергии активации,

- вычислим энергии активации глубоких центров по формуле (12).

Таким образом, находя энергию активации при различных напряжениях смещения и температурах, можно 
примерно выделить области, где преобладает рекомбинация через тот либо иной центр. Такая диаграмма построена на рис. 6. Рекомбинация через уровни с энергией активации $0.55 \mathrm{eV}$ преобладает в двух областях при низких температурах и малых смещениях и при высоких температурах и высоких смещениях. В оставшейся области рекомбинация преобладает через уровни с энергиями активации 0.39 и $0.45 \mathrm{eV}$. Такое поведение рекомбинационных потоков не является случайным. Для уровня $0.55 \mathrm{eV}$ в указанных областях время жизни быстро падает и он перехватывает на себя рекомбинационные потоки. В соответствии с рис. 6 время жизни при рекомбинации через уровни 0.39 и $0.45 \mathrm{eV}$ в области малых смещений быстро растет. Это обусловлено тем, что в этой области напряжений уровень инжекции еще мал, и процессы эмиссии с данных центров преобладают над процессами захвата.

\section{Заключение}

Таким образом, анализ, проведенный в настоящей работе, показывает, что эффективность преобразования исследуемых структур определяется процессом рекомбинации при малом уровне инжекции. В этом случае время жизни носителей заряда в приборе и его ток насыщения определяются рекомбинацией в области пространственного заряда.

Разработана методика анализа рекомбинационных процессов в этой области, которая позволяет определить рекомбинационные параметры центров в объеме полупроводника. Показано, что в рекомбинации участвуют три центра рекомбинации с энергиями активации 0.55 , 0.45 и $0.39 \mathrm{eV}$. Первый из них может быть связан с остаточными примесями золота либо железа в высокоомном кремнии. Этот центр обладает сильной температурной зависимостью скорости рекомбинации, которая может быть связана с особенностями температурного поведения скоростей захвата электронов и дырок на этот центр рекомбинации. Построена диаграмма, которая позволяет оценить, при каких условиях рекомбинационные потоки направляются на определенные центры. Эти исследования помогают оценить изменения в эффективности преобразования с изменениями условий протекания данного процесса.

Работа подготовлена при поддержке Минобрнауки РФ в рамках Государственной поддержки научных исследований.

\section{Список литературы}

[1] Flicker H., Loferski J.J., Elleman T.S. // IEEE T. Electron. Dev. 1964. N 2. P. 367-374.

[2] Kosteski T., Knerani N.P., Stradins P., Gaspari F. // IEE P. Circ. Dev. Syst. 2003. Vol. 150. N 4. P. 274-279.

[3] Guo H., Lal A. // IEEE Transducer. 2003. Vol. 36. P. $1122-1129$.
[4] Sun W., Kherani N.P. // Adv. Mater. 2005. Vol. 17. N 10. P. 1230-1237.

[5] Eiting C.J., Krishnamoorthy V., Rodgers S., George T. // Appl. Phys. Lett. 2006. Vol. 88. P. $064101-064110$.

[6] Chu J., Piao X. // J. Micro Nanolith. MEM. 2009. Vol. 8. P. $021180-021188$.

[8] Резнев А.А., Пустовалов А.А., Максимов Е.М., Передерий Н.P., Петренко Н.С. // Нано-микросистемная техника. 2009. № 3. С. 14-16.

[9] 3и С. Физика полупроводниковых приборов. М.: Мир, 1984. $455 \mathrm{c}$.

[10] Polymers, phosphors, and voltaics for radioisotope microbatteries. / Ed. by K.E. Bower, Y.A. Barbanel, Y.G.S.W. Bohnert. London; NY; Washington: CRC PRESS, 2002. 472 p.

[11] Булярский C.В., Грушко Н.С. Генерационно-рекомбинационные процессы в активных элементах. М.: МГУ, 1995. $399 \mathrm{c}$.

[12] Булярский С.В., Грушко Н.С., Сомов А.И., Лакалин А.В. // ФТП. 1997. Т. 31. С. 1146-1150.

[13] Булярский С.В., Грушко Н.С., Лакалин А.В. // Заводская лаборатория. 1997. Т. 63. С. 25-30. 